\title{
Resistance to Plasmodiophora brassicae in Brassica rapa and Brassica juncea genotypes From China
}

Hui Zhang, The Institute of Vegetables and Flowers, Chinese Academy of Agricultural Sciences, Beijing 100081, and Department of Agricultural, Food and Nutritional Science, University of Alberta, Edmonton, Alberta T6G 2P5, Canada; Jie Feng, Crop Diversification Centre North, Alberta Agriculture and Rural Development, Edmonton, Alberta T5Y 6H3, Canada; Shujiang Zhang, Shifan Zhang, Fei Li, The Institute of Vegetables and Flowers, Chinese Academy of Agricultural Sciences, Beijing; Stephen E. Strelkov, Department of Agricultural, Food and Nutritional Science, University of Alberta, Edmonton; Rifei Sun, The Institute of Vegetables and Flowers, Chinese Academy of Agricultural Sciences, Beijing 100081; and Sheau-Fang Hwang, Crop Diversification Centre North, Alberta Agriculture and Rural Development, Edmonton

\begin{abstract}
Zhang, H., Feng, J., Zhang, S., Zhang, S., Li, F., Strelkov, S. E., Sun, R., and Hwang, S.-F. 2015. Resistance to Plasmodiophora brassicae in Brassica rapa and Brassica juncea genotypes From China. Plant Dis. 99:776-779.

Clubroot disease, caused by Plasmodiophora brassicae Woronin, has become a major problem in cruciferous crops worldwide. Chinese cabbage (Brassica rapa), pak choi (B. rapa), and mustard (B. juncea) are important vegetable crops in China. Development of clubroot-resistant cultivars of these crops is urgently needed. In this study, $71 B$. rapa and $B$. juncea genotypes from China, including cultivars and inbred lines, were evaluated for resistance to three $P$. brassicae pathotypes. A significant interaction was observed between the P. brassicae pathotypes and the Brassica genotypes. Pathotype 3 , as defined on the differentials of Williams, exhibited the

weakest virulence on all plant material. By contrast, pathotypes 5 and 6 were both highly pathogenic on most of the tested genotypes. In all, 10 of the 14 Chinese cabbage cultivars were resistant to all three pathotypes, while 4 were resistant only to a specific pathotype. Seven of eight progenies obtained from the selfing of Chinese cabbage cultivars were resistant to pathotype 3 but most were susceptible to pathotypes 5 and 6 . Most inbred lines of Chinese cabbage and all inbred lines of pak choi and mustard were susceptible to all three pathotypes but their susceptibility was lower to pathotype 3 than to pathotypes 5 and 6 .
\end{abstract}

Clubroot, caused by the obligate parasite Plasmodiophora brassicae Woronin, has become a major problem in the production of cruciferous vegetable and oilseed crops worldwide (13). The pathogen has a complex life cycle comprising three main stages: survival as resting spores in the soil, primary infection of host root hairs, and secondary infection and development within the root cortex, which results in production of clubroot symptoms and new resting spores (14). The spores in the soil have a half-life of approximately 4 years and can remain viable for many years (30). As a result of $P$. brassicae infection, the roots of susceptible plants develop large clubs, which interfere with normal root function and result in the stunting, discoloration, and wilting of the aboveground parts of the hosts. Ultimately, crop yield and quality can be severely reduced (6). In China, clubroot was identified as an important quarantine target in the first National Plant Quarantine Conference in 1953 (31). It is prevalent in southern and western China $(17,36)$ and, at present, is distributed across most regions of the country, with average yield losses estimated at 20 to $30 \%$ in Chinese cabbage (32).

$P$. brassicae is known to consist of numerous physiological races or pathotypes that differ in their pattern of infection on host differentials. Numerous sets of differential hosts have been proposed to identify pathotypes of $P$. brassicae, with the differential system of Williams (34) being one of the most commonly used (25). These differentials consist of two cabbage (Brassica oleracea var. capitata) and two rutabaga (B. napus var. napobrassica) genotypes. According to the reactions on the Williams differentials, pathotype

Corresponding authors: R. Sun; E-mail: sunrifei@caas.cn; and S.-F. Hwang; E-mail: sheau-fang.hwang@gov.ab.ca

*The $\boldsymbol{e}$-Xtra logo stands for "electronic extra" and indicates that one supplementary table is published online.

Accepted for publication 7 November 2014.

http://dx.doi.org/10.1094/PDIS-08-14-0863-RE

(C) 2015 The American Phytopathological Society
4 is most common in China, representing nearly $80 \%$ of the characterized populations (3). In Alberta, Canada, pathotype 3 is predominant, although pathotypes $2,5,6$, and 8 are also present $(26,35)$

Many strategies have been proposed for the management of clubroot $(12,20)$, among which the cropping of resistant cultivars is believed to be most effective (23). Genetic resistance to clubroot can vary from broad spectrum to pathotype specific $(22,23)$, and both single resistance genes and quantitative trait loci (QTL) have been reported $(11,18,21,28,33)$. Cruciferous species have been extensively screened for clubroot resistance in Europe and North America, with a focus on the testing of cabbage, kale, cauliflower, broccoli, and canola $(2,9)$.

Over the past decade, many Asian Brassica cultivars have been imported to Europe and North America, and vice versa (15). Asian Brassica genotypes were evaluated for clubroot resistance by inoculation with Asian strains of $P$. brassicae $(8,16,37)$. Although this approach is justified, given that host genotypes grown in Asia are most likely to be exposed to these strains in the field, it is also important to evaluate Asian genotypes for resistance against strains of the pathogen of diverse geographic origins. This will facilitate the management of clubroot in two respects: (i) by preventing the introduction of host genotypes into areas that are dominated by virulent local strains of $P$. brassicae, and (ii) by providing information on new sources of resistance. This becomes more important given the increased trade and the globalization of agriculture. In the present study, $71 \mathrm{~B}$. rapa and $B$. juncea genotypes, including cultivars and inbred lines, were evaluated for resistance to three $P$. brassicae pathotypes (i.e., pathotypes 3, 5, and 6) from Canada, where clubroot has recently emerged as a major concern in canola and appears to be spreading rapidly (25).

\section{Materials and Methods}

Plant materials and preparation of seedlings. In total, $73 \mathrm{Bras}$ sica genotypes were included in the evaluation of clubroot resistance, including two canola cultivars that served as resistant ('45H29') and susceptible ('45H26') controls. The remaining 71 genotypes were 
divided into five groups based on their genetic makeup. Group 1 consisted of 14 commercial cultivars of Chinese cabbage (B. rapa $\mathrm{L}$. subsp. pekinensis (Lour.) Olsson) that are resistant to clubroot and currently are the most widely cultivated cultivars in China. Group 2 consisted of eight lines each derived from three generation of selfing of a Chinese cabbage resistant cultivar. Groups 3, 4, and 5 consisted of 26, 11, and 12 regular inbred lines of Chinese cabbage, pak choi (B. rapa L. subsp. chinensis (L.) Hanelt.), and mustard (B. juncea (L.) Czen and Coss), respectively, which represent the major cultivars of their corresponding species. Detailed information on all of the genotypes is listed in Supplementary Table S1.

To prepare the seedlings for inoculation, seed were surfacedisinfected in $1 \%$ sodium hypochlorite for $5 \mathrm{~min}$, washed with deionized water, and germinated in petri dishes $(9 \mathrm{~cm}$ in diameter) on moistened filter paper for 7 days. The resulting seedlings were used in all inoculation experiments as described below.

$P$. brassicae single-spore isolates and preparation of resting spores. Three $P$. brassicae strains from Canada-SACANSS1, ORCA SS4 and AbotJE-SS1, belonging to pathotypes 3, 5, and 6, respectively-were studied in these experiments (35). These strains were maintained at $-20^{\circ} \mathrm{C}$ as clubbed roots on the susceptible Chinese cabbage 'Granaat'. The resting spore inoculum was prepared by following a protocol modified after Feng et al. (7). Clubs were homogenized in $10 \%$ sucrose in a blender. The slurry was passed through eight layers of cheesecloth and the suspension was centrifuged at $50 \times g$ for $5 \mathrm{~min}$. The supernatant was transferred to a new tube and centrifuged at 2,000 $\times g$ for $5 \mathrm{~min}$. The resulting pellet consisted of two distinct layers: the lower black layer consisted of soil particles and the upper white to brownish layer of resting spores. The upper layer was resuspended in $5 \mathrm{ml}$ of water by gentle pipetting and transferred into a new tube containing $40 \mathrm{ml}$ of water. After centrifugation at $2,000 \times g$ for $5 \mathrm{~min}$, the supernatant was removed. Resting spores in the pellet were adjusted to a concentration $1 \times 10^{8}$ resting spores $/ \mathrm{ml}$ and surface disinfected by incubating with colistin sulfate (Sigma-Aldrich, Oakville, ON, Canada) at $1 \mu \mathrm{g} / \mathrm{ml}$ and vancomycin hydrochloride (Sigma-Aldrich) at $1 \mu \mathrm{g} / \mathrm{ml}$ at $25^{\circ} \mathrm{C}$ in the dark for $24 \mathrm{~h}$. The spores were used as inoculum after removal of the antibiotics by washing twice with $40 \mathrm{ml}$ of deionized water.

Plant inoculation. The seedlings were inoculated with the resting spores $\left(1 \times 10^{7}\right.$ spores $\left./ \mathrm{ml}\right)$ of each pathotype by immersing the roots into the suspension for $20 \mathrm{~s}$ The plants were transplanted into autoclaved Sunshine mix number 4 potting medium (SunGro Horticulture, Seba Beach, AB, Canada) in plastic cups ( 6 by 6 by $6 \mathrm{~cm}$ ) with one plant per cup (for group 2) or plastic nursery trays ( 50 by 25 by $6 \mathrm{~cm}$ ) with five plants per tray (for the other groups). The cups and trays were kept on water-filled plates for 14 days, then transferred onto the benches and watered from above every 2 days. For each group against all the pathotypes, the trial was conducted in a split-plot design with 16 repetitions (cups) for group 2 or a split-plot design with 3 repetitions (trays) for the other groups in a greenhouse maintained at 24 and $18^{\circ} \mathrm{C}$ (day and night, respectively) with a 16-h photoperiod. The experiments were conducted twice with similar results.

Data collection. At 28 days after inoculation (dai), plants were dug out and roots were washed with tap water. The severity of club development on each plant was rated on a 0-to-3 scale (16), where $0=$ no clubbing, $1=$ one or few small clubs on the lateral roots, $2=$ moderate clubbing on the lateral, roots and $3=$ severe clubbing on the lateral roots or on the main root. For group 2, each plant was treated as an experimental unit and the disease ratings from the 16 plants were averaged. For groups 1, 3, 4, and 5, severity ratings for each experimental tray were converted to an index of disease (ID) according to the following Equation (26):

$$
\mathrm{ID}(\%)=(n \times 0+n \times 1+n \times 2+n \times 3) /(N \times 3) \times 100
$$

Where: $n$ is the number of plants in each class, $N$ is the total number of plants, and $0,1,2$, and 3 are the symptom severity classes. The experiment was conducted twice. Cultivars or lines with an ID value higher than 26 were considered to be susceptible to clubroot (25).

Statistics. The dataset for each group of genotypes against pathotypes was analyzed with SAS software version 9.2 (SAS Institute, Cary, NC). Residuals for ID were calculated using SAS PROC GLM. These residuals were used to estimate skewedness and kurtosis with SAS PROC UNIVARIATE and homogeneity of variance by PROC GLM. Based on the result from the testing of the homogeneity of variance, the data from all genotype groups were pooled and the pooled data were analyzed as a split-plot design.

\section{Results}

B. rapa cultivars. The level of resistance of cultivars in group 1 differed among the three pathotypes (Fig. 1). Ten cultivars were resistant to all three pathotypes. Among the resistant cultivars, CR04 was completely resistant to all pathotypes, with all tested plants showing a rating score of 0 . Complete resistance to certain but not all pathotypes also was observed on all genotypes except CR07. Susceptible reactions also were observed. For instance, genotype CR03 was susceptible to pathotypes 5 and 6, CR05 was susceptible to pathotype 6, and CR09 and CR11 were susceptible to pathotype 5. However, all four of these genotypes were completely resistant to pathotype 3 . The variation in resistance within group 1 indicates that the resistance in some of these cultivars is pathotype specific, suggesting the importance of pathotype determination before using resistant cultivars.

Selfed progenies of resistant $B$. rapa cultivars. The genotypes in group 2 were derived from clubroot-resistant cultivars by three generations of forced selfing. Disease reactions segregated among the progeny of each genotype: highly resistant and highly susceptible plants were observed in most genotypes (Fig. 2). If a mean disease rating of 1 was used as the threshold to differentiate resistant and susceptible genotypes, all of the genotypes except CR17 were resistant

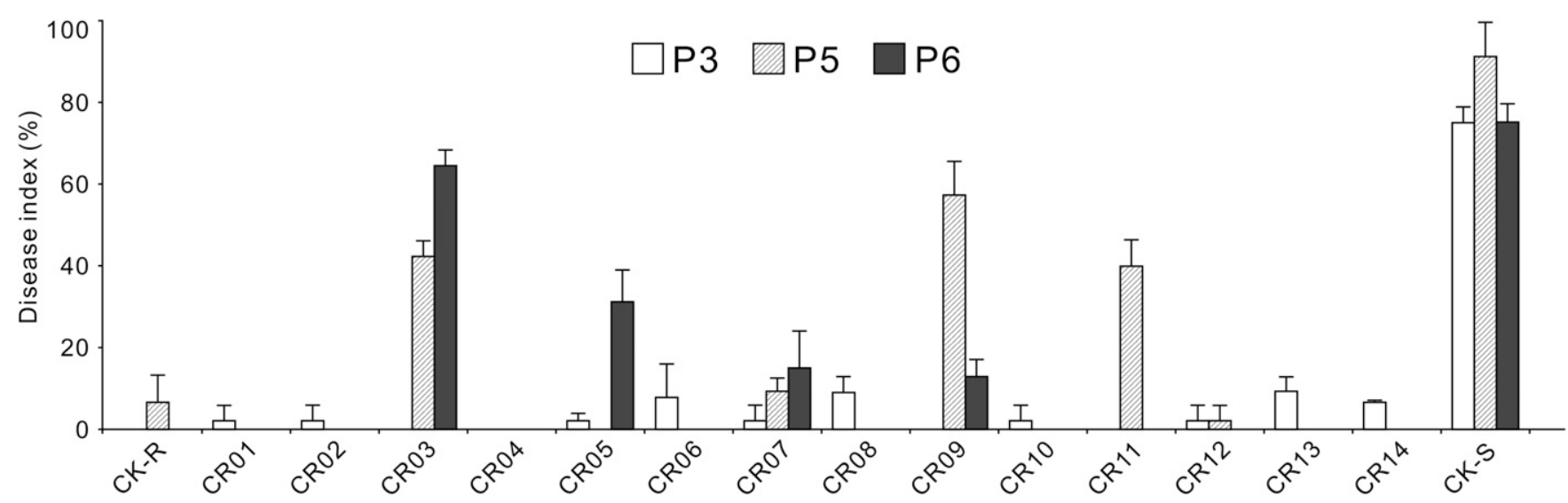

Fig. 1. Reactions of Chinese cabbage cultivars to pathotypes 3,5, and 6 of Plasmodiophora brassicae. 
to pathotype 3; CR20, CR21, and CR22 were resistant to pathotype 5; and CR18, CR21, and CR22 were resistant to pathotype 6. The higher level of resistance of the genotypes to pathotype 3 relative to pathotypes 5 and 6 indicated that resistance to pathotype 3 is under a more complete genetic control.

Brassica inbred lines. Groups 3, 4, and 5 consisted of inbred lines of Chinese cabbage, pak choi, and mustard, respectively. All of the Chinese cabbage inbred lines were susceptible to pathotype 6 (Fig. $3 \mathrm{~A})$ and most of them also were susceptible to pathotypes 3 and 5 . Resistance to pathotype 3 was present in CR31, CR42, and CR45, while resistance to pathotype 5 occurred in CR40. Compared with other Chinese cabbage inbred lines, CR40 showed lower levels of clubroot severity in response to inoculation with any of the three pathotypes (Fig. 3A). In groups 4 and 5, all of the inbred lines were susceptible to all three pathotypes (Fig. 3B and C).

Genotype-pathotype interactions. The Chinese cabbage genotypes, including resistant cultivars (group 1) and progenies of resistant cultivars (group 2), showed complete or partial resistance to

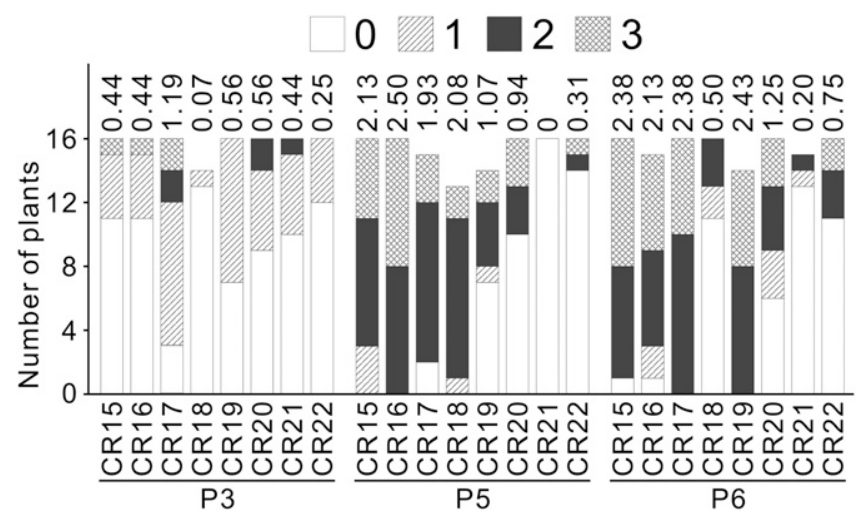

Fig. 2. Reactions of selfing progenies of Chinese cabbage cultivars to pathotypes 3,5 , and 6 of Plasmodiophora brassicae. The number of plants rated as 0 (no symptoms of clubroot), 1, 2, or 3 (heavy clubbing) on a clubroot severity scale is illustrated. The mean $(n=16)$ rating is indicated at the top of the plot. each of the three pathotypes. In contrast, most inbred lines of Chinese cabbage (group 3), pak choi (group 4), and mustard (group 5) were susceptible to clubroot. Based on all individuals from the five groups as a pool, analysis of variance indicated a highly significant interaction $(P<0.001)$ between $P$. brassicae pathotypes and Brassica genotypes. The virulence of pathotype 3 on the host genotypes was lower than pathotypes 5 and 6 , and pathotype 5 and 6 showed no difference in virulence.

\section{Discussion}

Resistance to clubroot has become an important objective in the breeding of cruciferous crops in China but the number of resistance sources is limited (3). In this study, most Chinese cabbage and pak choi and all of the mustard inbred lines were susceptible to pathotypes 3,5, and 6 of $P$. brassicae. The clubroot pathogen was introduced to China less than 200 years ago $(24,27)$; therefore, it is not surprising that few resources of resistance were identified in cruciferous crops from China.

Genetic resistance to clubroot has been reported in all of the major Brassica spp. except $B$. juncea and $B$. carinata (5). Most of the resistant genotypes identified to date appear to be pathotype specific, although some have shown a broader resistance spectrum $(4,29)$. The gene in 'Mendel' and 'Flower Nabana' conferred a dominant resistance to pathotype 3 (23). Resistance based on single genes is generally not durable, as was the case of a resistance breakdown in a Japanese Chinese cabbage cultivar caused by variation in the pathogenicity of $P$. brassicae (10). In the present study, resistance to pathotype 3 was better preserved than resistance to pathotypes 5 and 6 among the selfed progenies of resistant cultivars, indicating that genetic control of pathotype 3 resistance maybe more complex than the case of a single-gene segregating.

Wide genetic diversity has been reported to be present in the B genome of $B$. nigra $(9,19)$. Hasan et al. (9) reported a high level of resistance against five Canadian pathotypes of $P$. brassicae in the B-genome species B. nigra. Mustard also contains the B genome but no resistance was identified in the present or earlier studies. This may be explained by the possibility that the resistance in the B genome in $B$. juncea was lost during evolution because of a lack of selection pressure from the pathogen.
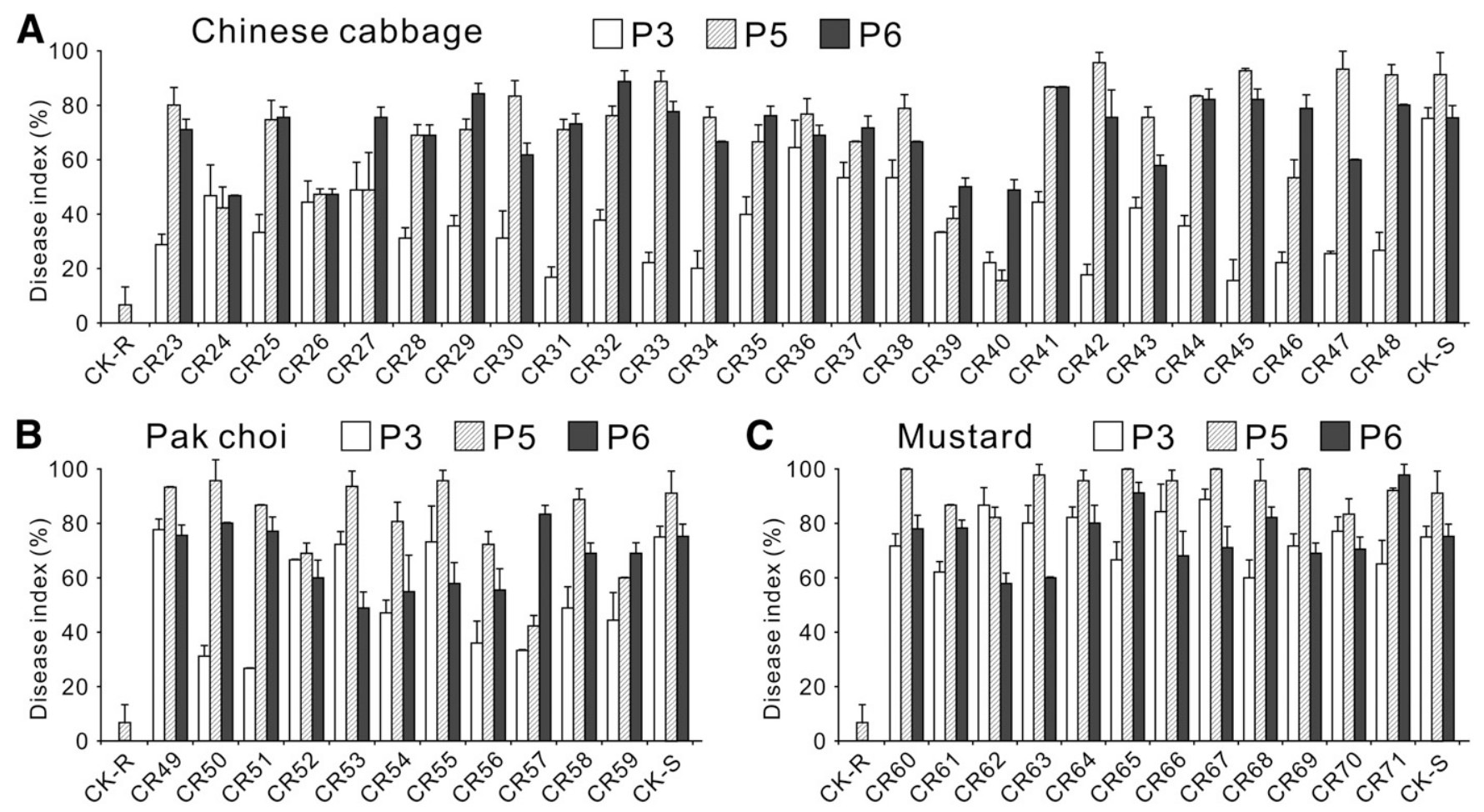

Fig. 3. Reactions of inbred lines of A, Chinese cabbage; B, pak choi; and C, mustard to pathotypes 3, 5, and 6 of Plasmodiophora brassicae. 
The present study identified a few lines of Chinese cabbage with high (e.g., CR04) or moderate resistance to pathotype 3. Pathotype 3 is the most prevalent and most virulent pathotype on canola in western Canada (1). Therefore, the genetic basis of resistance to pathotype 3 may warrant further study as a potential new resource for resistance breeding in Canada. Furthermore, this study also highlights the need to find sources of resistance to pathotypes 5 and 6 .

\section{Summary}

Most of the tested clubroot-resistant Chinese cabbage cultivars were resistant to Canadian pathotypes of $P$. brassicae. In Chinese cabbage, resistance to pathotype 3 was better preserved in the selfing progenies than resistance to pathotypes 5 and 6 . Some of the Chinese cabbage inbred lines were resistant to the Canadian pathotypes but none of the pak choi and mustard inbred lines was resistant to these pathotypes.

\section{Acknowledgments}

Funding support was provided by the National High Technology Research and Development Program ("863" Program) of China (2012AA100103002); China Agricultural Research System (CARS-25-A-01); National Key Technology R \& D Program from the Ministry of Science and Technology of China (2013 BAD01B04-03); Key Laboratory of Biology and Genetic Improvement of Horticultural Crops, Ministry of Agriculture, P. R. China; Alberta Agricultural and Rural Development, Canada; and the University of Alberta, Canada.

\section{Literature Cited}

1. Cao, T., Manolii, V. P., Strelkov, S. E., Hwang, S. F., and Howard R. J. 2009. Virulence and spread of Plasmodiophora brassicae (clubroot) in Alberta, Canada. Can. J. Plant Pathol. 31:321-329.

2. Carlsson, M., Bothmer, R. V., and Merker, A. 2004. Screening and evaluation of resistance to downy mildew (Peronospora parasitca) and clubroot (Plasmodiophora brassicae) in genetic resources of Brassica oleracea. Hereditas 141:293-300.

3. Chai, A. L., Xie, X. W., Shi, Y. X., and Li, B. J. 2014. Research status of clubroot (Plasmodiophora brassicae) on cruciferous crops in China. Can. J. Plant Pathol. 36(sup1):142-153.

4. Crute, I. R., Phelps, K., Barnes, A., Buczacki, S. T., and Crisp, P. 1983. The relationship between genotypes of three Brassica species and collections of Plasmodiophora brassicae. Plant Pathol. 32:405-420.

5. Diederichsen, E., Frauen, M., Linders, E. G. A., Hatakeyama, K., and Hirai, M. 2009. Status and perspectives of clubroot resistance breeding in crucifer crops. J. Plant Growth Regul. 28:265-281.

6. Dixon, G. R. 2009. The occurrence and economic impact of Plasmodiophora brassicae and clubroot disease. J. Plant Growth Regul. 28:194-202.

7. Feng J., Hwang, S. F., and Strelkov, S. E. 2013. Studies into primary and secondary infection processes by Plasmodiophora brassicae on canola. Plant Pathol. 62:177-183.

8. Geng, X. C., Shen, X. Q., Guo, Y. F., Ma, S. F., and Hong, Y. T. 2012. Breeding of anticlubroot AB lines type I of orange head Chinese cabbage. Acta Agric. Boreali-occidentalis Sin. 21:155-161.

9. Hasan, M. J., Strelkov, S. E., Howard, R. J., and Rahman, H. 2012. Screening of Brassica germplasm for resistance to Plasmodiophora brassicae pathotypes prevalent in Canada for broadening diversity in clubroot resistance. Can. J. Plant Sci. 92:501-515.

10. Hatakeyama, K., Fujimura, M., Ishida, M., Suzuki, T., and Sato, T. 2006. Classification of pathogenicity of Plasmodiophora brassicae field isolates in Japan based on resistance of F1 cultivars of Chinese cabbage (Brassica rapa L.) to clubroot. Acta Hortic. 706:323-328.

11. Hirai, M., Harada, T., Kubo, N., Tsukada, M., Suwabe, K., and Matsumoto, S. 2004. A novel locus for clubroot resistance in Brassica rapa and its linkage markers. Theor. Appl. Genet. 108:639-643.

12. Hwang, S. F., Howard, R. J., Strelkov, S. E., Gossen, B. D., and Peng, G. 2014. Management of clubroot (Plasmodiophora brassciae) on canola (Brassica napus) in western Canada. Can. J. Plant Pathol. 36:49-65.

13. Hwang, S. F., Stelkov, S. E., Feng, J., Gossen, B. D., and Howard, R. J. 2012. Plasmodiophora brassicae: A review of an emerging pathogen of the Canadian canola (Brassica napus) crop. Mol. Plant Pathol. 13:105-113.

14. Kageyama, K., and Asano, T. 2009. Life cycle of Plasmodiophora brassicae. J. Plant Growth Regul. 28:203-211.
15. Kershen, D. L. 2010. Trade and commerce in improved crops and food: An essay on food security. New Biotechnol. 27:623-627.

16. Kuginuki, Y., Yoshikawa, H., and Hirai, M. 1999. Variation in virulence of Plasmodiophora brassicae in Japan tested with clubroot resistant cultivars of Chinese cabbage (Brassica rapa L. ssp. pekinensis). Eur. J. Plant Pathol. 105:327-332.

17. Li, J. K., Bai, M., Xia, X. Y., Zhaxi, C. R., Ou, Z., and Wei, J. 1993. Research on the soil causes of clubroot on cruciferous vegetables in Yadong City. Tibet J. Tibet Univ. 8:70-72.

18. Nagaoka, T., Doulah, M. A. U., Matsumoto, S., Kawassaki, S., Ishikawa, T., Hori, H., and Okazaki, K. 2010. Identification of QTLs that control clubroot resistance in Brassica oleracea and comparative analysis of clubroot resistance genes between B. rapa and B. oleracea. Theor. Appl. Genet. 120: 1335-1346.

19. Negi, M. S., Sabharwal, V., Bhat, S. R., and Lakshmikumaran, M. 2004 Utility of AFLP markers for the assessment of genetic diversity within Brassica nigra germplasm. Plant Breed. 123:13-16.

20. Peng, G., Lahlali, R., Hwang, S. F., Pageau, D., Hynes, R. K., McDonald, M R., Gossen, B. D., and Strelkov, S. E. 2014. Crop rotation, cultivar resistance, and fungicides/biofungicides for managing clubroot (Plasmodiophora brassicae) on canola. Can. J. Plant Pathol. 36:99-112

21. Piao, Z. Y., Deng, Y. Q., Choi, S. R., Park, Y. J., and Lim, Y. P. 2004. SCAR and CAPS mapping of $C R b$, a gene conferring resistance to Plasmodiophora brassicae in Chinese cabbage (Brassica rapa ssp. pekinensis). Theor. Appl. Genet. 108:1458-1465.

22. Piao, Z. Y., Ramchiary, N., and Lim, Y. P. 2009. Genetic of clubroot resistance in Brassica species. J. Plant Growth Regul. 28:252-264.

23. Rahman, H., Peng, G., Yu, F., Falk, K. C., Kulkarni, M., and Selvaraj, G. 2014. Genetics and breeding for clubroot resistance in Canadian spring canola (Brassica napus L.). Can. J. Plant Pathol. 36:122-134.

24. Song, K. M., Osborn, T. C., and Williams, P. H. 1988. Brassica taxonomy based on nuclear restriction fragment length polymorphisms (RFLPs). I Preliminary analysis of subspecies within B. rapa (syn. campestris) and $B$. oleracea. Theor. Appl. Genet. 76:593-600.

25. Strelkov, S. E., and Hwang, S. F. 2014. Clubroot in the Canadian canola crop: 10 years into the outbreak. Can. J. Plant Pathol. 36(sup1):27-36.

26. Strelkov, S. E., Manolii, V. P., Cao, T., Xue, S., and Hwang, S. F. 2007 Pathotype classification of Plasmodiophora brassicae and its occurrence in Brassica napus in Alberta, Canada. J. Phytopathol. 155:706-712.

27. Sun, V. G. 1946. The evaluation of taxonomic characters of cultivated Brassica with a key to species and varieties. I. The characters. Bull. Torrey Bot. Club 73:244-281.

28. Suwabe, K., Tsukazaki, H., Iketani, H., Hatakeyama, K., Kondo, M., Fujimura, M., Nunome, T., Fukuoka, H., Hirai, M., and Matsumoto, S 2006. Simple sequence repeat-based comparative genomic between Brassica rapa and Arabidopsis thaliana: The genetic origin of clubroot resistance. Genetics 173:309-319.

29. Toxopeus, H., Dixon, G. R., and Mattusch, P. 1986. Physiological specialization in Plasmodiophora brassicae: An analysis by international experimentation. Trans. Br. Mycol. Soc. 87:279-287.

30. Wallenhammar, A. C. 1996. Prevalence of Plasmodiophora brassicae in a spring oilseed rape growing area in central Sweden and factors influencing soil infestation levels. Plant Pathol. 45:710-719.

31. Wang, H. F. 1962. Discussion of clubroot disease on cruciferous vegetables in China-from the disease found in Jiamusi City. J. Northeast Agric. Univ. 2: 47-48.

32. Wang, J., Huang, Y., Li, X. L., and Li, H. Z. 2011. Research progress in clubroot of crucifers. Plant Prot. 37:153-158.

33. Werner, S., Diederichsen, E., Frauen, M., Schondelmaier, J., and Jung, C. 2008. Genetic mapping of clubroot resistance genes in oilseed rape. Theor. Appl. Genet. 116:363-372.

34. Williams, P. H. 1966. A system for the determination of races of Plasmodiophora brassicae that infect cabbage and rutabaga. Phytopathology 56:624-626.

35. Xue, S., Cao, T., Howard, R. J., Hwang, S. F., and Strelkov, S. E. 2008 Isolation and variation in virulence of single-spore isolates of Plasmodiophora brassicae from Canada. Plant Dis. 92:456-462.

36. Yang, Y. H., Zhang, X. F., and Zou, L. J. 1992. Studies of the occurrence of Chinese cabbage clubroot in Changsha. J. Hunan Agric. Univ. 18: 854-858.

37. Zhang, T., Wu, D., Zhao, Z., Wang, Z., and Piao, Z. Y. 2012. Development of near isogenic lines for clubroot resistance in Chinese cabbage and their assessment. Mol. Plant Breed. 10:722-730. 